\title{
Pérdida prematura de dientes temporales en niños de 4 a 8 años que acuden a la Universidad Central del Ecuador
}

\author{
Premature loss of primary teeth in children aged 4 to 8 who attend the Central \\ University of Ecuador \\ María Espín-Flores ${ }^{1 a}$, Grace Revelo-Motta $^{1 b c d}$
}

\section{RESUMEN}

Objetivo: Determinar la frecuencia de la pérdida temprana de dientes deciduos de niños en edades de 4 a 8 que acudieron a la Universidad Central del Ecuador. Metodología. La muestra estuvo conformada por 453 historias clínicas de pacientes que fueron atendidos desde marzo del 2017 hasta abril del 2018. Se registraron los siguientes datos: edad, el género, pérdida dental prematura, diagnóstico del diente perdido y si se realizó el mantenedor de espacio. Se realizaron estadísticas descriptivas y las pruebas Welch Levene y ANOVA con un nivel de significancia del $5 \%(p<0.05)$. Resultados. La frecuencia de la pérdida prematura de dientes temporales fue del $56,5 \%$. sin diferencias estadísticas entre el género y la edad. El segundo molar inferior izquierdo fue el diente con mayor pérdida prematura $(7,3 \%)$. El mantenedor de espacio se realizó en el $30 \%$ de los casos. No hubo diferencias estadísticamente significativas por género y edad. Conclusión. La pérdida temprana de dientes deciduos fue elevada para el grupo estudiado. La realización del mantenedor de espacio fue baja. La implementación de programas educativos y preventivos para promover la salud oral pueden ayudar a los niños a mantener saludable la dentición temporal y prevenir alteraciones en el desarrollo de la oclusión.

Palabras clave: Diente Primario; Movimiento Mesial de los Dientes; Extracción Dental; Mantenimiento del Espacio en Ortodoncia; Caries Dental. (Fuente: DeCS BIREME)

\begin{abstract}
Objective: to determine the frequency of early loss of deciduous teeth in children aged 4 to 8 who attended the Central University of Ecuador. Methodology. The sample consisted of 453 medical records of patients who were seen from March 2017 to April 2018. The following data were recorded: age, gender, premature tooth loss, diagnosis of the missing tooth and whether the space maintainer was performed. Descriptive statistics and the Welch Levene and ANOVA tests were performed with a significance level of $5 \%(p<0.05)$. Results. The frequency of premature loss of primary teeth was $56.5 \%$. no statistical differences between gender and age. The lower left second molar was the tooth with the greatest premature loss $(7.3 \%)$. The space maintainer was performed in $30 \%$ of the cases. There were no statistically significant differences by gender and age. Conclution. Early loss of deciduous teeth was high for the group studied. Space maintainer realization was low. The implementation of educational and preventive programs to promote oral health can help children maintain healthy primary teeth and prevent alterations in the development of occlusion.
\end{abstract}

Key words: Tooth Deciduous; Mesial Movement of Teeth; Tooth Extraction; Space Maintenance; Orthodontic; Dental Caries. (Source: MeSH NLM)

${ }^{1}$ Facultad de Odontología, Universidad Central del Ecuador. Quito, Ecuador.

${ }^{\text {a Odontóloga. }}$

${ }^{\mathrm{b}}$ Especialista en Estomatología Pediátrica,

'Especialista en Ortodoncia y Ortopedia Maxilar.

d Docente.

Este es un artículo de acceso abierto distribuido bajo la licencia Creative Commons Atribución 4.0 Internacional (CC BY 4.0) https://creativecommons.org/licenses/by/4.0/deed.es

\section{Correspondencia:}

Grace Revelo Motta

Dirección: Av. Universitaria, Quito 170129. Ecuador.

Correo electrónico: gracerevelo@hotmail.com 


\section{INTRODUCCIÓN}

La dentición temporal, conocida también como primaria o decidua, es la primera que se establece en la cavidad oral. Los dientes temporales inician su erupción entre los 6 y 7 meses, completándose a los 29 meses de edad. Esta dentición es importante para el crecimiento y desarrollo craneofacial, porque mantiene el espacio para los dientes permanentes $y$ es fundamental para la masticación, la deglución, la articulación y la estética del niño ${ }^{(1)}$.

La pérdida prematura de dientes temporales (PPDT) se define de diversas maneras según la literatura publicada, se considera como perdida prematura, si se ha perdido el diente temporal: antes que el diente permanente sucesor se pueda palpar ${ }^{(2)}$, entre dos exámenes sucesivos en un periodo de un año ${ }^{(3)}$ y cuando fue extraído antes de la exfoliación natural del diente ${ }^{(4)}$.

La prevalencia de la PPDT varía en la población pediátrica dependiendo de diversos factores como: la fluoración del agua, nivel socioeconómico, prevalencia de caries dental y la filosofía de tratamiento del odontólogo ${ }^{(4)}$. La PPDT es un hallazgo común en la población infantil y los estudios han reportado rangos desde el 40 al $70 \%{ }^{(1,5-9)}$. Los efectos de la PPDT incluyen la alteración en la fonación con distorsión del habla ${ }^{(10)}$, cambios en la dimensión anterior y posterior de los arcos maxilares ${ }^{(11)}$, pérdida de espacio posterior a la extracción del diente temporal (12-14), linguoversión de los incisivos inferiores y sobrepase aumentado ${ }^{(15)} \mathrm{e}$ impacto en la calidad de vida ${ }^{(16)}$. La caries es el factor común para la PPDT, otros factores incluyen: desórdenes congénitos, exfoliación prematura de dientes temporales particularmente los caninos, erupción ectópica de dientes permanentes especialmente el primer molar, trauma dental y extracciones ortodónticas para tratamientos interceptivos ${ }^{(10,17-20)}$.

El objetivo de esta investigación fue determinar la frecuencia de la pérdida prematura de dientes temporales en niños de 4 a 8 años que acudieron a la Clínica de Odontopediatría de la Facultad de Odontología de la Universidad Central del Ecuador (FOUCE).

\section{METODOLOGIA}

Estudio descriptivo. Se revisaron 2355 historias clínicas de pacientes que acudieron a la Clínica de Odontopediatros desde marzo de 2017 hasta abril del 2018. La muestra fue de 453 expedientes clínicos de pacientes en edades de 4 a 8 que contaban con la fotocopia de la cédula del menor de edad y de su representante legal, se excluyeron las historias que no tuvieran la firma del docente inicial (autorización del tratamiento) y final (tratamiento realizado) del tratamiento de exodoncia.

Se realizó una clasificación según la edad, el género, diente temporal perdido, diagnóstico del diente y si se realizó el mantenedor de espacio. Para la identificación del diente perdido se usó el odontograma de Walter Drum utilizando dos números, el primero designa el cuadrante y el segundo el número del diente. El diagnóstico se determinó según el registro en la historia clínica basado en los códigos que se manejan en la clínica de odontopediatría de la FOUCE, se clasificaron como: caries, raíz abandonada, absceso/fístula, fractura dental y otros.

Los resultados se registraron en una hoja de cálculo de Microsoft Office Excel 2010. Para el análisis estadístico se empleó el programa SPSS versión 24.0, los datos se analizaron usando estadística descriptiva para obtener la frecuencia en valores absolutos y porcentajes. Las pruebas ANOVA y Welch Levene se aplicaron para verificar si existían diferencias estadísticamente significativas entre la pérdida prematura por la edad y el género con un nivel de significancia del $5 \%(p<0.05)$.

Consideraciones éticas. Por ser un estudio documental, en el cual no se mostraba la identidad de los pacientes, no se requirió la aprobacion de un comité de ética.

\section{RESULTADOS}

Del total de historias clínicas incluidas en el estudio, $256(56,5 \%)$ tuvieron pérdida prematura de dientes temporales, de estas historias 146 correspondían al género masculino y 110 al femenino y la mayor prevalencia se registró a los 8 años, no hubo diferencias estadísticamente significativas por género y edad (Tabla 1).

De las historias clínicas que registraron PPDT el diente 75 registró un mayor número de pérdidas $(12.89 \%)$ y el diagnóstico más frecuente fue el de caries dental $(62.89 \%)$ (Tabla 2$)$. Según la disposición de los dientes perdidos prematuramente, los molares (67\%) fueron los más afectados. Respecto al mantenedor de espacios se realizó en un porcentaje bajo $(20,31 \%)$ (Tabla 3). 
Tabla 1. Frecuencia y distribución por sexo y edad en relación con la pérdida temprana de dientes deciduos

\begin{tabular}{lccccccc}
\hline $\begin{array}{c}\text { Pérdida } \\
\text { prematura } \\
\text { de diente } \\
\text { temporal }\end{array}$ & $\begin{array}{c}\text { Femenino } \\
\mathbf{N}(\%)\end{array}$ & $\begin{array}{c}\text { Masculino } \\
\mathbf{N}(\%)\end{array}$ & $\begin{array}{c}\mathbf{4} \\
\mathbf{N}(\%)\end{array}$ & $\begin{array}{c}\mathbf{5} \\
\mathbf{N}(\%)\end{array}$ & $\begin{array}{c}\mathbf{6} \\
\mathbf{N}(\%)\end{array}$ & $\begin{array}{c}\mathbf{7} \\
\mathbf{N}(\%)\end{array}$ & $\begin{array}{c}\mathbf{8} \\
\mathbf{N}(\%)\end{array}$ \\
\hline $\mathbf{S i}$ & $110(24.22)$ & $146(32.22)$ & $7(1.54)$ & $43(9.49)$ & $64(14.12)$ & $49(10.81)$ & $93(20.52)$ \\
$\mathbf{N o}$ & $96(21.19)$ & $101(22.29)$ & $0(0.00)$ & $3(0.66)$ & $75(16.55)$ & $88(19.42)$ & $31(6.84)$ \\
Total & $206(45.47)$ & $247(54.52)$ & $7(1.54)$ & $46(10.15)$ & $139(30.68)$ & $137(30.24)$ & $124(27.37)$ \\
\hline
\end{tabular}

Tabla 2. Distribución de los dientes temporales que fueron afectados con la pérdida prematura según el diagnóstico dental

\begin{tabular}{ccccccc}
\hline & Caries & $\begin{array}{c}\text { Raíz } \\
\text { abandonada }\end{array}$ & $\begin{array}{c}\text { Diagnóstico dental } \\
\text { Absceso/Fístula }\end{array}$ & Fractura dental & Otro & Total \\
Diente & PPT, N (\%) & PPT, N (\%) & PPT, N (\%) & PPT, N (\%) & PPT, N (\%) & PPT, N (\%) \\
\hline $\mathbf{5 1}$ & $\mathbf{7}(2.73)$ & $4(1.56)$ & $1(0.39)$ & $1(0.39)$ & $3(1,17)$ & $16(6.25)$ \\
$\mathbf{5 2}$ & $4(1.56)$ & $2(0.78)$ & $1(0.39)$ & $0(0.00)$ & $2(0.78)$ & $9(3.51)$ \\
$\mathbf{5 3}$ & $0(0.00)$ & $0(0.00)$ & $0(0.00)$ & $0(0.00)$ & $0(0.00)$ & $0(0.00)$ \\
$\mathbf{5 4}$ & $13(5.07)$ & $5(1.95)$ & $2(0.78)$ & $3(1,17)$ & $2(0.78)$ & $25(9.75)$ \\
$\mathbf{5 5}$ & $5(1.95)$ & $1(0.39)$ & $0(0.00)$ & $0(0.00)$ & $2(0.78)$ & $8(3.12)$ \\
$\mathbf{6 1}$ & $3(1.17)$ & $7(2.73)$ & $1(0.39)$ & $1(0.39)$ & $0(0.00)$ & $12(4.68)$ \\
$\mathbf{6 2}$ & $5(1.95)$ & $0(0.00)$ & $0(0.00)$ & $1(0.39)$ & $0(0.00)$ & $6(2.34)$ \\
$\mathbf{6 3}$ & $0(0.00)$ & $0(0.00)$ & $0(0.00)$ & $0(0.00)$ & $0(0.00)$ & $0(0.00)$ \\
$\mathbf{6 4}$ & $22(8.59)$ & $1(0.39)$ & $1(0.39)$ & $0(0.00)$ & $2(0.78)$ & $26(10.15)$ \\
$\mathbf{6 5}$ & $14(5.46)$ & $2(0.78)$ & $0(0.00)$ & $0(0.00)$ & $0(0.00)$ & $16(6.25)$ \\
$\mathbf{7 1}$ & $3(1,17)$ & $2(0.78)$ & $0(0.00)$ & $1(0.39)$ & $2(0.78)$ & $8(3.12)$ \\
$\mathbf{7 2}$ & $5(1.95)$ & $2(0.78)$ & $0(0.00)$ & $0(0.00)$ & $1(0.39)$ & $8(3.12)$ \\
$\mathbf{7 3}$ & $2(0.78)$ & $0(0.00)$ & $0(0.00)$ & $0(0.00)$ & $1(0.39)$ & $3(1.17)$ \\
$\mathbf{7 4}$ & $18(7.03)$ & $4(1.56)$ & $0(0.00)$ & $0(0.00)$ & $1(0.39)$ & $23(8.98)$ \\
$\mathbf{7 5}$ & $22(8.59)$ & $5(1.95)$ & $2(0.78)$ & $1(0.39)$ & $3(1,17)$ & $33(12.89)$ \\
$\mathbf{8 1}$ & $4(1.56)$ & $2(0.78)$ & $0(0.00)$ & $1(0.39)$ & $2(0.78)$ & $9(3.51)$ \\
$\mathbf{8 2}$ & $5(1.95)$ & $0(0.00)$ & $0(0.00)$ & $2(0.78)$ & $1(0.39)$ & $8(3.12)$ \\
$\mathbf{8 3}$ & $3(1,17)$ & $0(0.00)$ & $0(0.00)$ & $0(0.00)$ & $1(0.39)$ & $4(1.56)$ \\
$\mathbf{8 4}$ & $16(6.25)$ & $4(1.56)$ & $0(0.00)$ & $1(0.39)$ & $2(0.78)$ & $23(8.98)$ \\
$\mathbf{8 5}$ & $10(3.90)$ & $6(2.34)$ & $1(0.39)$ & $0(0.00)$ & $2(0.78)$ & $19(7.42)$ \\
Total & $161(62.89)$ & $47(18.35)$ & $9(3.51)$ & $12(4.68)$ & $27(10.54)$ & $256(100)$ \\
\hline PPT & & & & & \\
& & & & & &
\end{tabular}

Tabla 3. Distribución de los molares perdidos prematuramente de acuerdo con el mantenimiento de espacios

\begin{tabular}{cccc}
\hline $\begin{array}{c}\text { Molar perdido } \\
\text { prematuramente }\end{array}$ & \multicolumn{2}{c}{ Mantenedor de espacio } & No \\
& $\mathbf{N ~ ( \% )}$ & $\mathbf{N ~ ( \% )}$ & $\begin{array}{c}\text { Total } \\
\mathbf{N}(\%)\end{array}$ \\
\hline $\mathbf{5 4}$ & $8(4.62)$ & $17(9.83)$ & $25(14.45)$ \\
$\mathbf{5 5}$ & $2(1.16)$ & $6(3.47)$ & $8(4.62)$ \\
$\mathbf{6 4}$ & $8(4.62)$ & $18(10.40)$ & $26(15.03)$ \\
$\mathbf{6 4}$ & $4(2.31)$ & $12(6.94)$ & $16(9.25)$ \\
$\mathbf{7 4}$ & $6(3.47)$ & $17(9.83)$ & $23(13.29)$ \\
$\mathbf{7 5}$ & $11(6.36)$ & $22(12.72)$ & $33(19.08)$ \\
$\mathbf{8 4}$ & $6(3.47)$ & $17(9.83)$ & $23(13.29)$ \\
$\mathbf{8 5}$ & $7(4.05)$ & $12(6.94)$ & $19(10.98)$ \\
Total & $52(30.06)$ & $121(69.94)$ & $173(100)$ \\
\hline
\end{tabular}




\section{DISCUSIÓN}

Los registros de las historias clínicas de niños de 4 a 8 años que fueron atendidos en la FOUCE indican una frecuencia de PPDT alta y no se encontraron diferencias por género y edad. De los molares perdidos prematuramente, un pequeño porcentaje tuvo mantenimiento de espacios. La alta frecuencia de PPDT (56\%) está de acuerdo con estudios actuales que se han realizado en Brasil, México, Yemen, Arabia Saudita, Alemania, Rumania, y Turquía ${ }^{(1,6-}$ $9,16,21,22)$.

La PPDT en el presente estudio estuvo representado por las historias clínicas de los niños que buscaron atención odontológica en la Clínica de Odontopediatría de la FOUCE. Por lo tanto, podemos esperar que estos niños presentan más necesidades de tratamiento dental que la población general. También puede ser porque los padres y los odontólogos no enfatizan en la importancia de la dentición temporal en la infancia. Estos padres y odontólogos pueden considerar que la prevención y el tratamiento de los dientes temporales no es importante porque estos dientes serán reemplazados ${ }^{(17,21)}$.

En este estudio no hubo diferencia estadísticamente significativa entre la PPDT entre niños y niñas. Este hallazgo fue similar a estudios anteriores, lo que implica que la pérdida prematura de dientes temporales se debe a un cuidado de la salud bucal deficiente, más que al género ${ }^{(1,21)}$. El mayor porcentaje de la PPDT fue a los 8 años y los molares se perdieron en mayor proporción que otros dientes temporales. El diente perdido con mayor frecuencia fue el segundo molar inferior izquierdo temporal, que es similar a los hallazgos de estudios anteriores $(1,21)$. Esto puede ser porque las fisuras se colonizan más fácilmente por S. mutans ${ }^{(23)}$. Los dientes inferiores presentan una mayor acumulación de placa en la región posterior, un mayor potencial de empaquetamiento de comida y el flujo salival disminuido por la zona vestibular

En este estudio, el mantenimiento de espacios posterior a la extracción se realizó en el $20.32 \%$ de los molares que tuvieron PPDT. La pérdida de espacios se evidencia a las tres semanas de la pérdida del segundo molar temporal, por ello, debe enfatizarse en el uso de mantenedores de espacios posterior a la extracción en un lapso de tiempo corto en los casos que presentan deficiencias severas en la longitud de arco ${ }^{(12)}$. La cantidad de espacio posterior a la pérdida del primer molar temporal, se disminuye con el tiempo y debe manejarse según las características del paciente, por ello, una buena práctica sugiere un manejo comprensivo de las características de cada caso antes de confirmar o no la necesidad de mantener el espacio ${ }^{(13)}$.

Es necesario enfatizar en la importancia de la salud bucal de los niños e informar a los padres sobre la probabilidad de problemas como maloclusiones, distorsión en el habla, impacto en la calidad de vida, entre otros, causados por la PPDT. El mantenimiento de espacios debe realizarse según las características individuales del paciente, en el caso de ser necesario, se dará una explicación a los padres sobre la función que ellos cumplen para que consideren realizarlo en un tiempo corto posterior a la extracción.

Este estudio tuvo como limitación que fue realizado en las historias clínicas de los niños que buscaron atención odontológica en la Clínica de Odontopediatría de Facultad de Odontología de la Universidad Central del Ecuador, por lo tanto, se puede esperar que estos niños presenten más necesidades de tratamiento dental que la población general. Por lo anterior, los datos de este estudio no pueden generalizarse a la población. Se requieren más estudios para abordar este problema en otras regiones del Ecuador y considerar otras variables como el nivel socioeconómico. La pérdida prematura de dientes temporales fue alta $(56.5 \%)$ y fue mayor a los ocho años. El segundo molar temporal inferior izquierdo fue el diente más afectado (12.89\%). El mantenedor de espacio se realizó en el $20.32 \%$ de los casos donde hubo pérdida del molar temporal.

Es imperativo concienciar a los niños y los padres, acerca del valor de la salud bucodental para mantener los dientes temporales y evitar las consecuencias de la pérdida temprana. Se debe orientar a los padres de los niños que tienen pérdida prematura de molares temporales, sobre el mantenimiento de espacios en caso de ser necesario.

Contribuciónes de autoría: MFEF y GERM diseñaron el estudio, recolectaron los datos y realizaron el procesamiento y análisis estadístico. La autoras redactaron el manuscrito y aprobaron la versión final del mismo. 
Fuente de financiamiento: El estudio fue financiado por las autoras.

Conflicto de intereses: Las autoras declararon no tener conflictos de interés.

\section{REFERENCIAS BIBLIOGRAFICAS}

1. Andronic A. Prevalence of early loss of primary teeth in 6-10 year old school children in Sibiu. Acta Medica Transilv [Internet]. 2017;22(4):128-9. Available from: http://www.amtsibiu.ro/index.php?option=com cont ent\&view=article\&id=3063:prevalence-of-earlyloss-of-primary-teeth-in-6-10-year-old-schoolchildren-in-sibiu\&catid=57:nr-4-2017

2. Björk A, Krebs A, Solow B. A method for epidemiological registration of malocculusion. Acta Odontol Scand. 1964;22(1):27-41.

3. Northway WM, Wainright RL, Demirjian A. Effects of premature loss of deciduous molars. Angle Orthod. 1984;54(4):295-329.

4. Bhujel N, Duggal MS, Saini P, Day PF. The effect of premature extraction of primary teeth on the subsequent need for orthodontic treatment. Eur Arch Paediatr Dent. 2016;17(6):423-34.

5. Pedersen J, Stensgaard K, Melsen B. Prevalence of malocclusion in relation to premature loss of primary teeth. Community Dent Oral Epidemiol. 1978;6(4):204-9.

6. Ceja S., Gómez G., Vargas N y, Pérez M. Pérdida prematura de dientes temporales en niños de cinco a 10 años que acuden a la Facultad de Odontología en Durango. Oral. 2019;20(62):16749.

7. Eroğlu CN, Boylu ÖF, Kurt M, Elasan S. Surgical evaluation of the early extraction of deciduous teeth by faculty of dentistry oral and maxillofacial surgery clinic throughout a year: Retrospective study. East J Med. 2018;23(3):182-6.

8. Al-Shahrani N, Al-Amri A, Hegazi F, Al-Rowis K, Al-Madani A, Hassan KS. The prevalence of premature loss of primary teeth and its impact on malocclusion in the Eastern Province of Saudi Arabia. Acta Odontol Scand. 2015;73(7):544-9.

9. Wagner $Y$, Knaup I, Knaup TJ, Jacobs C, Wolf M. Influence of a programme for prevention of early childhood caries on early orthodontic treatment needs. Clin Oral Investig. 2020;24(12):4313-24.

10. Nadelman P, Bedran N, Magno MB, Masterson D, de Castro ACR, Maia LC. Premature loss of primary anterior teeth and its consequences to primary dental arch and speech pattern: A systematic review and meta-analysis. Int $\mathrm{J}$ Paediatr Dent. 2020;30(6):687-712.

11. Lin YTJ, Lin YT. Long-term space changes after premature loss of a primary maxillary first molar. $J$ Dent Sci [Internet]. 2017;12(1):44-8. Available from: http://dx.doi.org/10.1016/j.jds.2016.06.005

12. Bindayel NA. Clinical evaluation of short term space variation following premature loss of primary second molar, at early permanent dentition stage. Saudi Dent J [Internet]. 2019;31(3):311-5. Available from: https://doi.org/10.1016/j.sdentj.2019.03.002

13. Kaklamanos EG, Lazaridou D, Tsiantou D, Kotsanos N, Athanasiou AE. Dental arch spatial changes after premature loss of first primary molars: a systematic review of controlled studies. Odontology. 2017;105(3):364-74.

14. Lucas-Rincón SE, Robles-Bermeo NL, LaraCarrillo E, Scougall-Vilchis RJ, Pontigo-Loyola AP, Rueda-lbarra $\mathrm{V}$, et al. Interproximal caries and premature tooth loss in primary dentition as risk factors for loss of space in the posterior sector: A cross-sectional study. Medicine (Baltimore). 2019;98(11):e14875.

15. Morera Pérez A, Ríos Paz Y, Villa Fernández D, Cobas A. Presencia de anomalías dentomaxilofaciales en niños con pérdida prematura de caninos temporales. MediSur. 2016;14(3):1-4.

16. Monte-Santo AS, Viana SVC, Moreira KMS, Imparato JCP, Mendes FM, Bonini GAVC. Prevalence of early loss of primary molar and its impact in schoolchildren's quality of life. Int $\mathrm{J}$ Paediatr Dent. 2018;28(6):595-601.

17. Bansal M, Gupta N, Gupta P, Arora V, Thakar S. Reasons for extraction in primary teeth among 512 years school children in Haryana, India- A cross-sectional study. J Clin Exp Dent. 2017;9(4):e545-9.

18. Nadelman $P$, Gárate KM, Oliveira A, Pithon MM, de Castro ACR, Maia LC. Dental arch perimeter changes as a result from premature loss of primary anterior teeth due to trauma: A case series in infant and pre-school children. Int J Paediatr Dent. 2020;

19. Costa VPP, Goettems ML, Baldissera EZ, Bertoldi AD, Torriani DD. Clinical and radiographic sequelae to primary teeth affected by dental trauma: a 9-year retrospective study. Braz Oral Res. 2016;30(1):1-9.

20. Vassiliou LV, Lalabekyan B, Jay A, Liew C, Whelan J, Newman L, et al. Head and neck sarcomas: A single institute series. Oral Oncol. 2017 Feb 1;65:16-22.

21. Murshid S, Al-Labani M, Aldhorae K, Rodis O. Prevalence of prematurely lost primary teeth in 510-year-old children in Thamar city, Yemen: A cross-sectional study. J Int Soc Prev Community Dent. 2016;6(8):S126-30.

22. López-Gómez SA, Villalobos-Rodelo JJ, ÁvilaBurgos L, Casanova-Rosado JF, Vallejos-Sánchez AA, Lucas-Rincón SE, et al. Relationship between premature loss of primary teeth with oral hygiene, consumption

of soft drinks, dental care, and previous caries experience. Sci Rep [Internet]. 2016;6(February):1-7. Available from: http://dx.doi.org/10.1038/srep21147 
23. Caufield PW, Cutter GR, Dasanayake AP. Initial Acquisition of Mutans Streptococci by Infants:
Evidence for a Discrete Window of Infectivity. J Dent Res. 1993;72(1):37-45.

María Espín-Flores

ORCID iD: (1) https://orcid.org/0000-0002-6026-3078

maife_ef25@hotmail.com

Grace Revelo-Motta

ORCID iD: (1) http://orcid.org/0000-0002-6448-0440

gracerevelo@hotmail.com

Copyright $\odot$ La revista. La revista Kiru es publicada por la Facultad de Odontología de la Universidad de San Martín de Porres, en Lima, Perú. 